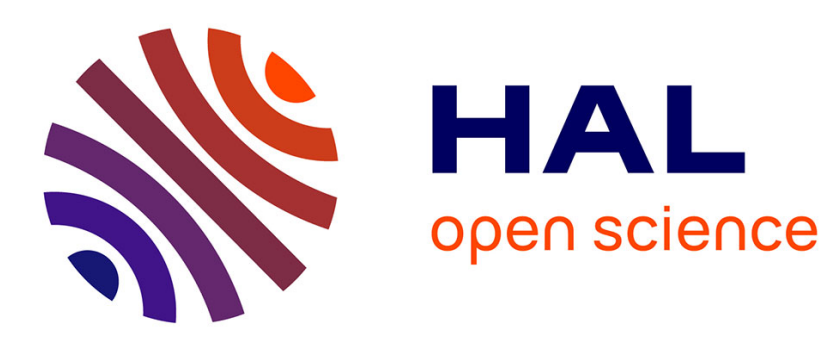

\title{
Reconstruction of the interface between two layered media using far field measurements
}

\author{
Lahcène Chorfi, Patricia Gaitan
}

\section{To cite this version:}

Lahcène Chorfi, Patricia Gaitan. Reconstruction of the interface between two layered media using far field measurements. Inverse Problems, 2011, 27 (7). hal-00516765

\section{HAL Id: hal-00516765 \\ https://hal.science/hal-00516765}

Submitted on 12 Sep 2010

HAL is a multi-disciplinary open access archive for the deposit and dissemination of scientific research documents, whether they are published or not. The documents may come from teaching and research institutions in France or abroad, or from public or private research centers.
L'archive ouverte pluridisciplinaire HAL, est destinée au dépôt et à la diffusion de documents scientifiques de niveau recherche, publiés ou non, émanant des établissements d'enseignement et de recherche français ou étrangers, des laboratoires publics ou privés. 


\title{
Reconstruction of the interface between two layered media using far field measurements
}

\author{
Lahcène Chorfi ${ }^{1}$ and Patricia Gaitan ${ }^{2}$ \\ ${ }^{1}$ Laboratoire LMA, Université Badji Mokhtar, Annaba, Algérie \\ ${ }^{2}$ Université d'Aix-Marseille, IUT Aix-en-Provence Avenue Gaston Berger 413 av \\ Gaston Berger, 13625 Aix-en-Provence et LATP, UMR CNRS 6632, 39, rue Joliot \\ Curie, 13453 Marseille Cedex 13, France
}

E-mail: 1_chorfi@hotmail.com and patricia.gaitan@univmed.fr

\begin{abstract}
We consider an inverse transmission scattering problem. This problem consists in determining an interface between two-layered media by farfield measurements. We prove that the interface is uniquely determined by the measurements of the far field pattern associated to incoming plane waves at a fixed frequency. For the reconstruction of the interface we solve a non linear integral equation using a truncated Newton-CG algorithm.
\end{abstract}

Keywords: Inverse Scattering, Interface Reconstruction, Far Field Pattern. 


\section{Introduction}

Inverse transmission problems of the Helmholtz equation are of fundamental interest due to the large range of applications: geophysical exploration, medical imaging, underwater acoustics, non-destructive testing. We are interested in the inverse problem to recover one-dimensional interface $\Gamma$ separating two dielectric (or acoustic) media $\Omega^{+}$and $\Omega^{-}$ with different wave numbers $k^{ \pm}$. We assume that $\Gamma$ is a graph of a smooth function $f$ with compact support, which means that $\Gamma=\Gamma^{\prime} \cup \gamma$ with $\gamma$ a local perturbation of the flat interface $\left\{x_{2}=0\right\}$ (see figure 1). More precisely our direct problem consists in finding two functions $u=\left(u^{+}, u^{-}\right)$that solve the Helmholtz equation

$$
\triangle u^{ \pm}+\left(k^{ \pm}\right)^{2} u^{ \pm}=0 \text { in } \Omega^{ \pm} .
$$

We assume that the scattered waves $u^{ \pm}$satisfy the Sommerfeld radiation condition in the domain $\Omega^{ \pm}$and that for a given incident plane wave $u^{i n c}$ the total field $u_{t o t}=u_{0}+u$, $u_{0}=\left(u^{i n c}+u_{r}, u_{t}\right)$, satisfies the transmission conditions

$$
u^{+}-u^{-}=-\left[u_{0}\right], \quad \frac{\partial}{\partial n}\left(u^{+}-u^{-}\right)=-\left[\frac{\partial u_{0}}{\partial n}\right] \quad \text { on } \Gamma,
$$

here $\left[u_{0}\right]=u^{i n c}+u_{r}-u_{t}$ where $u_{r}$ and $u_{t}$ denotes the reflected and transmitted waves by a flat interface according to the geometrical optic.

The inverse problem we are concerned, is to recover $\gamma$ (local perturbation) from the knowledge of wave numbers $\left(k^{+}, k^{-}\right)$and the far field pattern $u_{\infty}^{+}$of $u^{+}$for one incoming plane wave $u^{i n c}=e^{i k^{+} x \cdot d},|d|=1$. Introducing an operator $\mathcal{F}^{+}: f \rightarrow u_{\infty}^{+}$that maps an admissible parametrization $f$ of $\gamma$ onto the far field pattern $u_{\infty}^{+}$of scattered field $u^{+}$, this problem can formulated as an operator equation:

$$
\mathcal{F}^{+}(f)=u_{\infty}^{+} .
$$

Since inverse problem such as (1.1) is ill-posed, a regularization method has to be applied in order to obtain stable solution, especially if noisy data are used. We use a regularized Gauss-Newton method to find an approximation of $\gamma$ given some noisy data $u_{\infty}^{+, \delta}$, a bound $\delta$ of the error $\left\|u_{\infty}-u_{\infty}^{+, \delta}\right\|$ and an initial guess $\gamma_{0}$. Regularized Gauss-Newton method have been used for the approximate solution of inverse obstacle scattering problems for time harmonic waves in the case of smooth obstacles (see [17, 16, 19]) and crack detection [18].

In [2], the inverse scattering problem to recover the one-dimensional profile of a dielectric rough interface has been considered. The proposed method in [2] yields satisfactory reconstructions of interfaces having a perturbation less than one half of the wavelength. The level of roughness is small. The reconstruction is achieved via a single illumination by a plane wave at a fixed frequency and the reflected field measurements are performed on a line parallel to the surface. The data we use for our inverse problem are not the same (like in [2]) but our inversion algorithm can be applied to a wide range of frequencies, depending on the size of the roughness.

Our paper is organized as follow. In section 2, we set the direct problem, give a representation of the solution and deduce the far field pattern. In section 3 , we perform 
an asymptotic analysis of the Green function of the perturbed problem far away and near the interface. In section 4, we set our inverse problem, prove the uniqueness theorem and propose an iterative method to reconstruct the interface. In section 5, we present some numerical experiments which illustrate our algorithm.

Notations. We introduce the following notations. Let $\Omega^{+}$and $\Omega^{-}$be two regions of $\mathbb{R}^{2}$ defined by: $\Omega^{+}=\left\{x=\left(x_{1}, x_{2}\right) \in \mathbb{R}^{2}: x_{2}>f\left(x_{1}\right)\right\}$ and $\Omega^{-}=$ $\left\{x=\left(x_{1}, x_{2}\right) \in \mathbb{R}^{2}: x_{2}<f\left(x_{1}\right)\right\}$ where $f: \mathbb{R} \rightarrow \mathbb{R}$ is a continuous function such that $f \in C^{2}([-a, a])$ and $f(x)=0$ if $|x|>a$, for some $a>0$. Denote $\Gamma=\left\{\left(x_{1}, f\left(x_{1}\right)\right), x_{1} \in \mathbb{R}\right\}$ the boundary of $\Omega^{ \pm}$and $\gamma=\left\{\left(x_{1}, f\left(x_{1}\right)\right) \in \mathbb{R}^{2} ;-a<x_{1}<a\right\}$ is an open arc with $n(x)=\left(-f^{\prime}\left(x_{1}\right), \frac{1}{\sqrt{1+\left(f^{\prime}\left(x_{1}\right)\right)^{2}}}\right)$ the outgoing normal. The two unbounded domains $\Omega^{+}$and $\Omega^{-}$are acoustic media with wave numbers $k^{+}$and $k^{-}$. We set $k(x)=\left\{\begin{array}{l}k^{+} \text {if } x \in \Omega^{+}, \\ k^{-} \text {if } x \in \Omega^{-} .\end{array}\right.$. Denote $\mathbb{R}_{+}^{2}=\left\{x=\left(x_{1}, x_{2}\right) \in \mathbb{R}^{2} ; x_{2}>0\right\}$ and $\mathbb{R}_{-}^{2}=\left\{x=\left(x_{1}, x_{2}\right) \in \mathbb{R}^{2} ; x_{2}<0\right\}$.

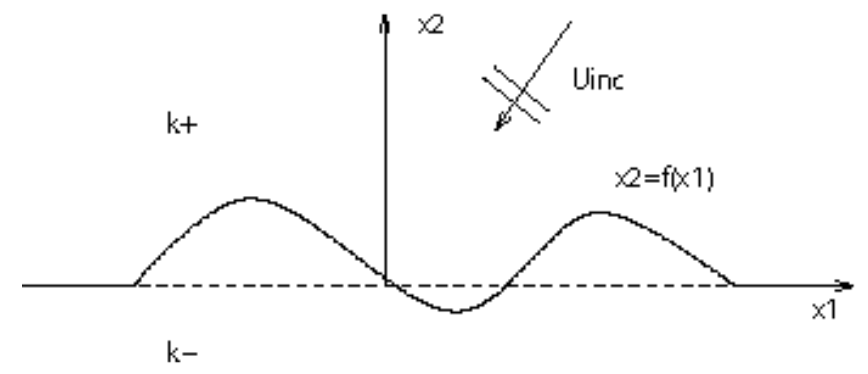

Figure 1. Geometry of the problem

\section{The transmission problem}

Let us give a precise definition of the direct problem. Let $u^{i n c}$ be an incident plane wave given by

$$
u^{i n c}(x)=\exp \left(-i k^{+}\left(x_{1} \cos \theta+x_{2} \sin \theta\right)\right), \quad \theta \in(0, \pi) .
$$

If the interface is flat i.e. $f=0$, the reflected wave $u_{r}$ and transmitted wave $u_{t}$ are given by Fresnel formula (see for example [10]):

$$
\begin{gathered}
u_{r}(x)=R(\theta) \exp \left(i k^{+}\left(-x_{1} \cos \theta+x_{2} \sin \theta\right)\right) \quad \text { for } x_{2}>0, \\
u_{t}(x)=T(\theta) \exp \left(-i\left(k^{+} x_{1} \cos \theta+k^{-} x_{2} \sin \chi\right)\right) \quad \text { for } x_{2}<0,
\end{gathered}
$$

with $\sin \chi=\sqrt{1-\nu^{2} \cos ^{2} \theta}, \nu=\frac{k^{+}}{k^{-}}$, and the reflection and transmission coefficients are given by

$$
R(\theta)=\frac{k^{+} \sin \theta-k^{-} \sin \chi}{k^{+} \sin \theta+k^{-} \sin \chi}, \quad T(\theta)=\frac{2 k^{+} \sin \theta}{k^{+} \sin \theta+k^{-} \sin \chi} .
$$


If $\Gamma$ is the perturbed interface $(f \neq 0)$, we introduce the function

$$
u_{0}(x)=\left\{\begin{array}{l}
u^{i n c}(x)+u_{r}(x) \text { in } \Omega^{+} \\
u_{t}(x) \text { in } \Omega^{-}
\end{array}\right.
$$

Here we used the analytic continuation of $u^{i n c}, u_{r}$ and $u_{t}$ to the whole plane $\mathbb{R}^{2}$.

Our scattering problem is the following: find $u$ such that the total field $u^{\text {tot }}=u_{0}+u$ satisfies the Helmholtz equation in $\mathbb{R}^{2}$ :

$$
\left\{\begin{array}{c}
\Delta u^{t o t}+k(x) u^{t o t}=0 \text { in } H_{l o c}^{1}\left(\mathbb{R}^{2}\right) \\
u \text { satisfies Sommerfeld radiation condition when }|x| \rightarrow+\infty .
\end{array}\right.
$$

If we denote $u^{ \pm}=\left.u\right|_{\Omega^{ \pm}}$, the problem (2.4) is equivalent to the transmission problem: find $u^{ \pm} \in C^{2}\left(\Omega^{ \pm}\right) \cap C^{1}\left(\bar{\Omega}^{ \pm}\right)$such that

$$
\left\{\begin{array}{l}
\Delta u^{+}+\left(k^{+}\right)^{2} u^{+}=0 \quad \text { in } \Omega^{+}, \\
\Delta u^{-}+\left(k^{-}\right)^{2} u^{-}=0 \quad \text { in } \Omega^{-}, \\
u^{+}-u^{-}=-u^{i n c}-u_{r}+u_{t} \quad \text { on } \gamma, \\
\frac{\partial u}{\partial n}\left(u^{+}-u^{-}\right)=-\frac{\partial}{\partial n}\left(u^{i n c}+u_{r}-u_{t}\right) \quad \text { on } \gamma, \\
u^{+}-u^{-}=0, \quad \frac{\partial u}{\partial n}\left(u^{+}-u^{-}\right)=0 \quad \text { on } \Gamma \backslash \gamma, \\
\frac{\partial u^{ \pm}}{\partial r}-i k^{ \pm} u^{ \pm}=o\left(\frac{1}{\sqrt{r}}\right), \quad \text { as } r=|x| \rightarrow+\infty \text { and } x \in \Omega^{ \pm} .
\end{array}\right.
$$

Remark 2.1 - The critical angle $\theta_{c} \in(0, \pi)$ is defined by $\cos \theta_{c}=\frac{k^{-}}{k^{+}}$if $k^{+} \geq k^{-}$ and $\theta_{c}=0$ if $k^{+}<k^{-}$. We can consider different cases:

1- If $\theta_{c}<|\theta|<\pi-\theta_{c}$, then $\sin \chi=\sqrt{1-\left(\frac{k^{+}}{k^{-}}\right)^{2} \cos ^{2} \theta} \in \mathbb{R}$ and $u_{t}$ is a plane wave.

2- If $0<|\theta|<\theta_{c}$ or $\pi-\theta_{c}<\theta<\pi$ then $\sin \chi=i \sqrt{1-\left(\frac{k^{+}}{k^{-}}\right)^{2} \cos ^{2} \theta} \in i \mathbb{R}$ and $u_{t}$ is a guided wave by the interface $\left\{x_{2}=0\right\}$.

3- If $\theta=\theta_{c}$ or $\theta=\pi-\theta_{c}$ then $\chi=0$ and $u_{t}$ a grazing ray.

- If $u^{i n c}(x)=\exp \left(-i k^{-}\left(x_{1} \cos \theta+x_{2} \sin \theta\right)\right)$ with $\theta \in(-\pi, 0)$, then interchanging $k^{+}$ and $k^{-}$, we obtain formula similar to (2.2)-(2.3).

\subsection{Representation of the scattered field}

For the resolution of (2.5) we use the integral method related with the Green's function $G(x, y)$ of the two-layered medium, i.e. fundamental solution of the unperturbed problem $(f=0)$. The function $G(x, y)$ is represented as the inverse Fourier transform of it's spectral image (see $[14,9]$ ). Indeed, for an observation point $x=\left(x_{1}, x_{2}\right)$ and a source point $y=\left(y_{1}, y_{2}\right) \in \mathbb{R}_{+}^{2}$, the Green's function is given by

$$
G(x, y)=\left\{\begin{array}{ccc}
G_{0}(x, y)+G_{r}(x, y) & \text { if } & x \in \mathbb{R}_{+}^{2}, \\
G_{t}(x, y) & \text { if } & x \in \mathbb{R}_{-}^{2},
\end{array}\right.
$$

with

$$
G_{0}(x, y)=\frac{i}{4} H_{0}^{(1)}\left(k^{+}|x-y|\right)
$$




$$
G_{t}(x, y)=\frac{i}{2 \pi} \int_{-\infty}^{+\infty} \frac{e^{i\left(-\beta x_{2}+\alpha y_{2}\right)}}{\alpha+\beta} e^{i \xi\left(x_{1}-y_{1}\right)} d \xi
$$

and

$$
G_{r}(x, y)=\frac{i}{4 \pi} \int_{-\infty}^{+\infty} \frac{\alpha-\beta}{\alpha(\alpha+\beta)} e^{i \alpha\left(x_{2}+y_{2}\right)} e^{i \xi\left(x_{1}-y_{1}\right)} d \xi
$$

where $\alpha=\sqrt{\left(k^{+}\right)^{2}-\xi^{2}}$ and $\beta=\sqrt{\left(k^{-}\right)^{2}-\xi^{2}}$ (if $|\xi|>k^{ \pm}$we choose the determination $\left.\sqrt{\left(k^{ \pm}\right)^{2}-\xi^{2}}=i \sqrt{\xi^{2}-\left(k^{ \pm}\right)^{2}}\right)$. If $y_{2}<0$ similar formula are available. The following result holds:

Theorem 2.2 The problem (2.5) has an unique solution represented by the combined simple and double-layer potentials:

$$
u(x)=\int_{\gamma}\left(G(x, y)\left[\frac{\partial u_{0}}{\partial n}\right]+\frac{\partial G(x, y)}{\partial n(y)}\left[u_{0}\right]\right) d s(y), \quad x \in \mathbb{R}^{2} \backslash \gamma .
$$

If we write this solution in the form $u=D\left[u_{0}\right]+S\left[\frac{\partial u_{0}}{\partial n}\right]$, then the operator

$$
(g, h) \mapsto D g+S h
$$

is bounded from $\tilde{H}^{\frac{1}{2}}(\gamma) \times \tilde{H}^{-\frac{1}{2}}(\gamma)$ to $H_{l o c}^{1}\left(\mathbb{R}^{2}\right)$.

Sketch of the proof: For the representation (2.6) we refer to the report [3] where the author generalizes the trace formulas, well known for the classical potentials, to the potentials $S$ and $D([7])$. Indeed, the singularity of $G(x, y)$ looks like those of $G_{0}(x, y)$ (see Appendix A1). This solution is unique by a Rellich type theorem (see Theorem 2.5). Recall the definitions of Sobolev spaces $\tilde{H}^{\frac{1}{2}}(\gamma)=\left\{v \in H^{\frac{1}{2}}(\Gamma) ; \operatorname{supp} v \subset \bar{\gamma}\right\}$ and $\tilde{H}^{-\frac{1}{2}}(\gamma):=\left(\tilde{H}^{\frac{1}{2}}(\gamma)\right)^{\prime}$ the dual space, then the continuity of the operators $S$ et $D$ between theses spaces and $H_{l o c}^{1}\left(\mathbb{R}^{2}\right)$ can be established using the same arguments as in $[4,5]$ for the crack problem.

\subsection{Far Field Pattern}

2.2.1. Asymptotic behavior at infinity The objective here is to provide the asymptotic behavior of the solution $u(x)$, obtained in Theorem 2.2, when $r=|x| \rightarrow+\infty$. For this we need the asymptotic behavior of the Green function.

When the source point is in the upper half space $\left(y_{2}>0\right)$ and for observation points in the lower half space $\left(x_{2}<0\right)$, we have the asymptotic formula for $G_{t}(x, y)$ (see [14]):

$$
G_{t}(x, y)=\frac{e^{i\left(k^{-} r+\frac{\pi}{4}\right)}}{2 \sqrt{2 \pi k^{-} r}} S_{t}(\phi) e^{i \widetilde{x} y}+O\left(\frac{1}{r}\right), \quad \phi \in[-\pi, 0]
$$

with

$$
\begin{gathered}
S_{t}(\phi)=\frac{2 k^{-} \sin \phi}{k^{-} \sin \phi-\sqrt{\left(k^{+}\right)^{2}-\left(k^{-}\right)^{2} \cos ^{2} \phi}}, \\
x=r(\cos \phi, \sin \phi), \quad \widetilde{x}=\left(-k^{-} \cos \phi, \sqrt{\left(k^{+}\right)^{2}-\left(k^{-}\right)^{2} \cos ^{2} \phi}\right) .
\end{gathered}
$$


For $x_{2}>0$ and $y_{2}>0$ we have

$$
G(x, y)=\frac{e^{i\left(k^{+} r+\frac{\pi}{4}\right)}}{2 \sqrt{2 \pi k^{+} r}} e^{-i k^{+} \hat{x} y}+\frac{e^{i\left(k^{+} r+\frac{\pi}{4}\right)}}{2 \sqrt{2 \pi k^{+} r}} S_{r}(\phi) e^{i k^{+} \widetilde{x} y}+O\left(\frac{1}{r}\right), \phi \in[0, \pi],
$$

with

$$
S_{r}(\phi)=\frac{\alpha_{s}-\beta_{s}}{\alpha_{s}+\beta_{s}}, \quad \alpha_{s}=k^{+} \sin \phi, \quad \beta_{s}=\sqrt{\left(k^{-}\right)^{2}-\left(k^{+}\right)^{2} \cos ^{2} \phi}
$$

and

$$
x=r(\cos \phi, \sin \phi), \quad \hat{x}=(\cos \phi, \sin \phi), \tilde{x}=(-\cos \phi, \sin \phi) .
$$

These formula imply the asymptotic behavior:

$$
u(x)=\frac{e^{i\left(k^{+} r+\frac{\pi}{4}\right)}}{2 \sqrt{2 \pi k^{+} r}} A^{+}(\theta, \phi)+\frac{e^{i\left(k^{-} r+\frac{\pi}{4}\right)}}{2 \sqrt{2 \pi k^{-r}}} A^{-}(\theta, \phi)+O\left(\frac{1}{r}\right) .
$$

Definition 2.3 The pair $u_{\infty}(\theta, \phi)=\left(A^{+}(\theta, \phi), A^{-}(\theta, \phi)\right)$ called the far field pattern is defined by:

(i) For $\phi \in[0, \pi]$

$$
\begin{aligned}
A^{+}(\theta, \phi) & =\int_{\gamma}\left(\left[\frac{\partial u_{0}}{\partial n}\right] e^{-i k^{+} \hat{x} y}+\left[u_{0}\right] \frac{\partial e^{-i k^{+} \hat{x} y}}{\partial n}\right) d s(y) \\
& +S_{r}(\phi) \int_{\gamma}\left(\left[\frac{\partial u_{0}}{\partial n}\right] e^{i k^{+} \widetilde{x} y}+\left[u_{0}\right] \frac{\partial e^{i k^{+} \widetilde{x} y}}{\partial n}\right) d s(y), \\
A^{-}(\theta, \phi) & =0
\end{aligned}
$$

where

$$
\begin{gathered}
S_{r}(\phi)=\frac{\alpha_{s}-\beta_{s}}{\alpha_{s}+\beta_{s}}, \quad \alpha_{s}=k^{+} \sin \phi, \quad \beta_{s}=\sqrt{\left(k^{-}\right)^{2}-\left(k^{+}\right)^{2} \cos ^{2} \phi}, \\
\hat{x}=(\cos \phi, \sin \phi) \text { and } \widetilde{x}=(-\cos \phi, \sin \phi) .
\end{gathered}
$$

(ii) For $\phi \in[\pi, 0]$

$$
\begin{aligned}
& A^{+}(\theta, \phi)=0 \\
& A^{-}(\theta, \phi)=S_{t}(\phi) \int_{\gamma}\left(\left[\frac{\partial u_{0}}{\partial n}\right] e^{i \tilde{x} y}+\left[u_{0}\right] \frac{\partial e^{i \tilde{x} y}}{\partial n}\right) d s(y),
\end{aligned}
$$

where

$$
S_{t}(\phi)=\frac{2 k^{-} \sin \phi}{k^{-} \sin \phi-\sqrt{\left(k^{+}\right)^{2}-\left(k^{-}\right)^{2} \cos ^{2} \phi}}, \tilde{x}=\left(-k^{-} \cos \phi, \sqrt{\left(k^{+}\right)^{2}-\left(k^{-}\right)^{2} \cos ^{2} \phi}\right) .
$$

Remark 2.4 All these formula are available even if the incident angle $\theta \in[-\pi, 0]$.

Theorem 2.5 Let $u^{+}$and $u^{-}$satisfying

$$
\left\{\begin{array}{l}
\Delta u^{+}+\left(k^{+}\right)^{2} u^{+}=0 \quad \text { for } x \in \mathbb{R}_{+}^{2} \text { and }|x|>R_{0} \\
\Delta u^{-}+\left(k^{-}\right)^{2} u^{-}=0 \quad \text { for } x \in \mathbb{R}_{-}^{2} \quad \text { and }|x|>R_{0} \\
u^{+}=u^{-} ; \quad \frac{\partial u^{+}}{\partial x_{2}}=\frac{\partial u^{-}}{\partial x_{2}}, \quad \text { if } x_{2}=0 \text { and }\left|x_{1}\right|>R_{0} \\
\frac{\partial u^{ \pm}}{\partial r}-i k^{ \pm} u^{ \pm}=o\left(\frac{1}{\sqrt{r}}\right) \quad \text { as } r=|x| \rightarrow+\infty \quad \text { and } \quad x \in \mathbb{R}_{ \pm}^{2} .
\end{array}\right.
$$


Moreover, if we assume that

$$
\lim _{R \rightarrow+\infty} \int_{\Sigma^{ \pm}(R)}\left|u^{ \pm}\right|^{2} d s=0
$$

where $\Sigma^{ \pm}(R)=\left\{x \in \mathbb{R}_{ \pm}^{2},|x|=R\right\}$, then $u^{ \pm}(x)=0$ for $|x|>R_{0}$.

\section{Proof:}

This result is another version of the classical Rellich theorem. The proof follows from the uniqueness theorem obtained by Kristensson [20], which is the generalization of the result of Odeh [21].

Theorem 2.6 Let $u=\left(u^{+}, u^{-}\right)$be a solution of (2.5). We assume that the far field pattern vanishes, that is $A^{+}(\theta, \phi)=A^{-}(\theta, \phi)=0$, for all $\theta, \phi \in[-\pi, \pi]$. Then $u(x)=0$ in $\mathbb{R}^{2}$.

\section{Proof:}

From (2.9), we have

$$
\int_{\Sigma_{R}}|u|^{2} d s=\frac{1}{8 \pi k^{+}} \int_{0}^{\pi}\left|A^{+}(\theta, \phi)\right|^{2} d \phi+\frac{1}{8 \pi k^{-}} \int_{-\pi}^{0}\left|A^{-}(\theta, \phi)\right|^{2} d \phi+O\left(\frac{1}{\sqrt{R}}\right) .
$$

If $u_{\infty}(\theta, \phi) \equiv(0,0)$, the result follows from Theorem 2.5 and from an unique continuation principle. This principle is proved in [7] for smooth solution (in $C^{2}$ ) but is actually available for generalized solution (in $H_{l o c}^{2}$ ).

2.2.2. Operators $\mathcal{F}^{ \pm} \quad$ Suppose that the incident angle $\theta \in(0, \pi)$ is fixed and consider the amplitude $A^{+}(\theta, \phi)$ (resp. $A^{-}(\theta, \phi)$ ) which is the far field pattern when the observation point is in the upper half space $\Omega^{+}$i.e $\phi \in[0, \pi]:=s^{+}$(resp. in the lower half space $\Omega^{-}$, i.e $\phi \in[-\pi, 0]=s^{-}$), (see Definition 2.3). Note that in the geophysical exploration it is more reasonable to consider the observation angle in $s^{+}$. In this section we explicit the dependence of $A^{ \pm}(\theta, \phi)$ as a function of the parametrization $f$ of $\gamma$. Indeed we obtain a non linear mapping $\mathcal{F}^{ \pm}: f \longrightarrow g$ such that

$$
g(\phi)=\int_{-a}^{a} F^{ \pm}\left(\phi, y_{1}, f\left(y_{1}\right), f^{\prime}\left(y_{1}\right)\right) d y_{1}, \quad \phi \in s^{ \pm} .
$$

\section{Observation angle in $(0, \pi)$}

Recall that for $\phi \in] 0, \pi[$,

$$
\begin{aligned}
A^{+}(\theta, \phi) & =\int_{\gamma}\left(\left[\frac{\partial u_{0}}{\partial n}\right] e^{-i k^{+} \hat{x} y}+\left[u_{0}\right] \frac{\partial e^{-i k^{+} \hat{x} y}}{\partial n}\right) d s(y) \\
& +S_{r}(\phi) \int_{\gamma}\left(\left[\frac{\partial u_{0}}{\partial n}\right] e^{i k^{+} \widetilde{x} y}+\left[u_{0}\right] \frac{\partial e^{i k^{+} \tilde{x} y}}{\partial n}\right) d s(y),
\end{aligned}
$$


where

$$
\begin{gathered}
S_{r}(\phi)=\frac{\alpha_{s}-\beta_{s}}{\alpha_{s}+\beta_{s}}, \quad \alpha_{s}=k^{+} \sin \phi, \quad \beta_{s}=\sqrt{\left(k^{-}\right)^{2}-\left(k^{+}\right)^{2} \cos ^{2} \phi}, \\
\hat{x}=(\cos \phi, \sin \phi) \text { and } \widetilde{x}=(-\cos \phi, \sin \phi) .
\end{gathered}
$$

The jumps of $u_{0}$ and $\frac{\partial u_{0}}{\partial n}$ are given by:

$$
\begin{aligned}
{\left[u_{0}\right]=u^{i n c}+u_{r}-u_{t}=} & \exp \left[-i k^{+}\left(y_{1} \cos \theta+y_{2} \sin \theta\right)\right]+R(\theta) \exp \left[-i k^{+}\left(y_{1} \cos \theta-y_{2} \sin \theta\right)\right] \\
& -T(\theta) \exp \left[-i\left(k^{+} y_{1} \cos \theta+k^{-} y_{2} \sin \chi\right)\right] .
\end{aligned}
$$

Using the rule:

$$
\frac{\partial g(y)}{\partial n} d s(y)=\left[\frac{\partial g(y)}{\partial y_{1}}\left(-f^{\prime}\left(y_{1}\right)\right)+\frac{\partial g(y)}{\partial y_{2}}\right] d y_{1} \quad \text { where } y_{2}=f\left(y_{1}\right),-a<y_{1}<a,
$$

we obtain

$$
\left[\frac{\partial u_{0}}{\partial n}\right]=\frac{\partial\left[u_{0}\right]}{\partial n}=-f^{\prime}\left(y_{1}\right)\left(\frac{\partial\left(u_{r}+u^{i n c}\right)}{\partial y_{1}}-\frac{\partial u_{t}}{\partial y_{1}}\right)+\left(\frac{\partial\left(u^{i n c}+u_{r}\right)}{\partial y_{2}}-\frac{\partial u_{t}}{\partial y_{2}}\right) .
$$

Then

$$
\begin{gathered}
{\left[\frac{\partial u_{0}}{\partial n}\right]=i k^{+} f^{\prime}\left(y_{1}\right) \cos \theta\left(u^{i n c}+u_{r}-u_{t}\right)-i k^{+} \sin \theta\left(u^{i n c}-u_{r}\right)+i k^{-} \sin \chi u_{t}} \\
\frac{\partial e^{-i k^{+} \hat{x} y}}{\partial n} d s=i k^{+}\left(f^{\prime}\left(y_{1}\right) \cos \phi-\sin \phi\right) e^{-i k^{+} \hat{x} y} d y_{1}
\end{gathered}
$$

and

$$
\frac{\partial e^{i k^{+} \widetilde{x} y}}{\partial n} d s=i k^{+}\left(f^{\prime}\left(y_{1}\right) \cos \phi+\sin \phi\right) e^{i k^{+} \widetilde{x} y} d y_{1} .
$$

The equation (2.10) can be written:

$$
\begin{aligned}
A^{+}(\theta, \phi) & =\int_{-a}^{a}\left[i k^{+}\left(\cos \theta f^{\prime}\left(y_{1}\right)+\cos \phi f^{\prime}\left(y_{1}\right)-\sin \phi\right)\left(u^{i n c}+u_{r}-u_{t}\right)(2.11)\right. \\
& \left.-i k^{+} \sin \theta\left(u^{i n c}-u_{r}\right)+i k^{-} \sin \chi u_{t}\right] e^{-i k^{+} \hat{x} y} d y_{1} \\
& +S_{r}(\phi) \int_{-a}^{a}\left[i k^{+}\left(\cos \theta f^{\prime}\left(y_{1}\right)+\cos \phi f^{\prime}\left(y_{1}\right)+\sin \phi\right)\left(u^{i n c}+u_{r}-u_{t}\right)\right. \\
& \left.-i k^{+} \sin \theta\left(u^{i n c}-u_{r}\right)+i k^{-} \sin \chi u_{t}\right] e^{i k^{+} \tilde{x} y} d y_{1}
\end{aligned}
$$

which is of the form:

$$
A^{+}(\theta, \phi)=\int_{-a}^{a} F^{+}\left(\theta, \phi, y_{1}, f\left(y_{1}\right), f^{\prime}\left(y_{1}\right)\right) d y_{1},
$$

with

$F^{+}\left(\theta, \phi, y_{1}, f\left(y_{1}\right), f^{\prime}\left(y_{1}\right)\right)=e^{-b_{1} y_{1}}\left[\left(\left(a_{1}+b_{1}\right)\left(u^{i n c}+u_{r}-u_{t}\right) f^{\prime}\left(y_{1}\right)-a_{2}\left(u^{i n c}-u_{r}\right)+c_{1} u_{t}\right)\left(e^{-b_{2} f\left(y_{1}\right)}\right.\right.$ 
Reconstruction of the interface between two layered media

$$
\left.\left.+S_{r}(\phi) e^{b_{2} f\left(y_{1}\right)}\right)-b_{2}\left(u^{i n c}+u_{r}-u_{t}\right)\left(e^{-b_{2} f\left(y_{1}\right)}-S_{r}(\phi) e^{b_{2} f\left(y_{1}\right)}\right)\right],
$$

where

$$
\begin{aligned}
& a_{1}=i k^{+} \cos \theta, \quad a_{2}=i k^{+} \sin \theta, \\
& b_{1}=i k^{+} \cos \phi, \quad b_{2}=i k^{+} \sin \phi, \quad c_{1}=i k^{-} \sin \chi .
\end{aligned}
$$

More precisely we obtain the expression

$$
\begin{aligned}
F^{+}\left(\theta, \phi, y_{1}, f\left(y_{1}\right), f^{\prime}\left(y_{1}\right)\right. & =F_{1}^{+}\left(\theta, \phi, y_{1}, f\left(y_{1}\right), f^{\prime}\left(y_{1}\right)\right) \\
& +S_{r}(\phi) F_{2}^{+}\left(\theta, \phi, y_{1}, f\left(y_{1}\right), f^{\prime}\left(y_{1}\right)\right)
\end{aligned}
$$

where

$$
\begin{aligned}
& F_{1}^{+}\left(\theta, \phi, y_{1}, f\left(y_{1}\right), f^{\prime}\left(y_{1}\right)\right)=\left(A_{1} f^{\prime}\left(y_{1}\right)+B_{1}\right) \exp \left(-A_{1} y_{1}-B_{1} f\left(y_{1}\right)\right) \\
&+ R(\theta)\left(A_{1} f^{\prime}\left(y_{1}\right)+B_{2}\right) \exp \left(A_{1} y_{1}+B_{2} f\left(y_{1}\right)\right) \\
&+ T(\theta)\left(-A_{1} f^{\prime}\left(y_{1}\right)+B_{3}\right) \exp \left(-A_{1} y_{1}-B_{3} f\left(y_{1}\right)\right), \\
& F_{2}^{+}\left(\theta, \phi, y_{1}, f\left(y_{1}\right), f^{\prime}\left(y_{1}\right)\right)=\left(A_{1} f^{\prime}\left(y_{1}\right)-B_{2}\right) \exp \left(A_{2} y_{1}-B_{1} f\left(y_{1}\right)\right) \\
&+R(\theta)\left(A_{1} f^{\prime}\left(y_{1}\right)+B_{1}\right) \exp \left(A_{2} y_{1}+B_{2} f\left(y_{1}\right)\right) \\
&+T(\theta)\left(-A_{1} f^{\prime}\left(y_{1}\right)+B_{4}\right) \exp \left(A_{1} y_{1}-B_{3} f\left(y_{1}\right)\right), \\
& A_{1}=a_{1}+b_{1}, A_{2}=-a_{1}+b_{1}, B_{1}=a_{2}+b_{2}, B_{2}=a_{2}-b_{2} \\
& B_{3}=c_{1}+b_{2}, B_{4}=c_{1}-b_{2} .
\end{aligned}
$$

\section{Observation angle in $(-\pi, 0)$}

For $\phi \in]-\pi, 0[$ we have

$$
A^{-}(\theta, \phi)=S_{t}(\phi) \int_{\gamma}\left(\left[\frac{\partial u_{0}}{\partial n}\right] e^{i \tilde{x} y}+\left[u_{0}\right] \frac{\partial e^{i \tilde{x} y}}{\partial n}\right) d s(y),
$$

where

$$
S_{t}(\phi)=\frac{2 k^{-} \sin \phi}{k^{-} \sin \phi-\sqrt{\left(k^{+}\right)^{2}-\left(k^{-}\right)^{2} \cos ^{2} \phi}}, \quad \widetilde{x}=\left(-k^{-} \cos \phi, \sqrt{\left(k^{+}\right)^{2}-\left(k^{-}\right)^{2} \cos ^{2} \phi}\right) .
$$

As in the previous case the integral equation (2.16) take the form:

$$
u_{\infty}^{-}(\theta, \phi)=\int_{-a}^{a} F^{-}\left(\theta, \phi, y_{1}, f\left(y_{1}\right), f^{\prime}\left(y_{1}\right)\right) d y_{1}
$$

with

$$
\begin{aligned}
F^{-}\left(\theta, \phi, y_{1}, f\left(y_{1}\right), f^{\prime}\left(y_{1}\right)\right)=S_{t}(\phi) & {\left[\left(f^{\prime}\left(y_{1}\right)\left(a_{1}+a_{3}\right)+i \sqrt{\left(k^{+}\right)^{2}+a_{3}^{2}}\right)\left(u^{i n c}+u_{r}-u_{t}\right)\right.} \\
& \left.-a_{2}\left(u^{i n c}-u_{r}\right)+c_{1} u_{t}\right] e^{-a_{3} y_{1}+i \sqrt{\left(k^{+}\right)^{2}+a_{3}^{2}} f\left(y_{1}\right)}
\end{aligned}
$$

Which can be written as

$$
\begin{array}{r}
F^{-}\left(\theta, \phi, y_{1}, f\left(y_{1}\right), f^{\prime}\left(y_{1}\right)\right)=S(\phi)\left[\left(C_{1} f^{\prime}\left(y_{1}\right)+C_{2}\right) R(\theta) \exp \left(-C_{1} y_{1}+C_{2} f\left(y_{1}\right)\right)+\right. \\
\left(-C_{1} f^{\prime}\left(y_{1}\right)+C_{4}\right) T(\theta) \exp \left(-C_{1} y_{1}-C_{4} f\left(y_{1}\right)\right)+ \\
\left.\left(C_{1} f^{\prime}\left(y_{1}\right)+C_{3}\right) \exp \left(-C_{1} y_{1}+C_{3} f\left(y_{1}\right)\right)\right]
\end{array}
$$


with

$$
\begin{aligned}
& a_{3}=i k^{-} \cos \phi, \quad C_{1}=a_{1}+a_{3}, \quad C_{2}=a_{2}+\sqrt{\left(k^{+}\right)^{2}+a_{3}^{2}}, \\
& C_{3}=-a_{2}+\sqrt{\left(k^{+}\right)^{2}+a_{3}^{2}}, \quad C_{4}=c_{1}-\sqrt{\left(k^{+}\right)^{2}+a_{3}^{2}} .
\end{aligned}
$$

\section{Perturbed Green function $\mathcal{G}(x, y)$}

In this section, we consider the Green function $\mathcal{G}(x, y)$ of the perturbed layered medium. $\mathcal{G}(x, y)$ satisfies $\Delta \mathcal{G}(x, y)+k^{2}(x) \mathcal{G}(x, y)=\delta(x-y)$ in $D^{\prime}\left(\mathbb{R}^{2}\right)$ and the radiation condition. We can write $\mathcal{G}(x, y)=G(x, y)+\mathcal{G}^{(s)}(x, y)$ where $\mathcal{G}^{(s)}$ solves the transmission problem $(2.5)$ with $u_{0}(x)=G(x, y)$. Such a function exists, is unique, and is represented by the potentials (see (2.6))

$$
\mathcal{G}^{(s)}(x, y)=\int_{\gamma}\left(G(x, z)\left[\frac{\partial G(z, y)}{\partial n(z)}\right]+\frac{\partial G(x, z)}{\partial n(z)}[G(z, y)]\right) d s(z), x \in \mathbb{R}^{2} \backslash \gamma
$$

We point out that $G(z, y)$ is defined as follows, for $y \in \Omega^{ \pm}$

$$
G(z, y)=\left\{\begin{array}{ccc}
G_{0}\left(k^{ \pm} ; z, y\right)+G_{r}(z, y) & \text { if } & z \in \Omega^{ \pm} \\
G_{t}(z, y) & \text { if } & z \in \Omega^{ \pm}
\end{array}\right.
$$

In this last definition we consider the analytic continuation of $G_{r}$ and $G_{t}$ in the whole space $\mathbb{R}^{2}$ (see Appendix A1).

\subsection{Far-field}

Using the asymptotic formulas of $G_{r}(x, y)$ and $\left.G_{t}(x, y)\right)$, when $|y| \rightarrow+\infty$, and the representation (3.1) we obtain the asymptotic formulas

Proposition 3.1 Suppose that $x_{2}>0$ and $y=r(\cos \theta, \sin \theta)$. Then we have for $r \rightarrow+\infty$ :

1- if $\theta \in(0, \pi)$,

$$
\mathcal{G}^{(s)}(x, y)=c \frac{e^{i k^{+} r}}{\sqrt{k^{+} r}}(u(x,-\theta)+u(x, \theta))+O\left(\frac{1}{r}\right), \quad\left(c=\frac{e^{i \pi / 4}}{2 \sqrt{2 \pi}}\right),
$$

2- if $\theta \in(-\pi, 0)$,

$$
\mathcal{G}^{(s)}(x, y)=c \frac{e^{i k^{-} r}}{\sqrt{k^{-} r}}(u(x,-\theta)+u(x, \theta))+O\left(\frac{1}{r}\right),
$$

where $u(x, \theta)$ is the scattered wave corresponding to the incident wave $u^{i n c}(\theta)=$ $e^{-i k^{+}\left(x_{1} \cos \theta+x_{2} \sin \theta\right)}$ for $\theta \in(0, \pi)$ (respectively $u^{i n c}(\theta)=e^{-i k^{-}\left(x_{1} \cos \theta+x_{2} \sin \theta\right)}$ for $\theta \in(-\pi, 0))$.

Theses relations are similar to the mixed reciprocity relations well known in the obstacle problem [7]. 


\subsection{Singularities}

The aim in this section is to recover the singularities of $\mathcal{G}^{(s)}(x, y)$ when $x$ and $y$ are close to the interface $\gamma$. For this we begin with by the following lemma.

Lemma 3.2 Recall that $G_{0}(k, y, z):=\frac{i}{4} H_{0}^{(1)}(k|y-z|)$ is the fundamental solution of the Helmholtz equation in $\mathbb{R}^{2}$. We have the following asymptotic expansions as $|y-z| \rightarrow 0$ :

(1) $G_{0}(k, y, z)=\frac{-1}{2 \pi}(\log |y-z|+C)+O\left(|y-z|^{2} \log |y-z|\right), \quad C=C_{E}+\log \frac{k}{2}-i \frac{\pi}{2}, \quad C_{E} \simeq$ 0.5772,

(2) $\frac{\partial G_{0}(k, y, z)}{\partial z_{j}}=-\frac{1}{2 \pi} \frac{y_{j}-z_{j}}{|y-z|^{2}}+O(|y-z| \log |y-z|)$,

(3) If $\gamma \in C^{2}$ and $z \in \gamma$, then $\frac{\partial G_{0}(k, y, z)}{\partial n(z)}=-\frac{1}{2 \pi|y-z|}+O(|y-z| \log |y-z|)$.

\section{Proof:}

- Items (1) and (2) follow from the asymptotic expansion of Hankel functions ([1]):

$$
H_{0}^{(1)}(t)=\frac{2 i}{\pi}\left(\log t+C_{E}\right)+1+O\left(t^{2} \log t\right), \quad \frac{d}{d t} H_{0}^{(1)}(t)=-H_{1}^{(1)}(t)=\frac{2 i}{\pi t}+O(t \log t),
$$

when $t \rightarrow 0$, where $C_{E} \simeq 0.5772$ is the Euler's constant.

- For $z \in \gamma, \frac{\partial G_{0}(k, y, z)}{\partial n(z)}=-\frac{\langle y-z, n(z)\rangle}{|y-z|^{2}}+O(|y-z| \log |y-z|)$. On the other hand, if $\gamma$ is $C^{2}$, we can establish the behavior $\frac{\langle y-z, n(z)\rangle}{|y-z|}=1+O\left(z_{1}\right)$, and item $(3)$ follows.

Assume that $t \in \gamma$, let $B(t)=B(t, \delta)$ be a small ball centered at the point $t$, $y=t+n(t) y_{2}$ with $0<y_{2}<\delta$ and $x=y$. We introduce a local coordinate system $\left(z_{1}, z_{2}\right)$ in $B(t)$ with the origin at $t$ and such that $z_{2}=0$ is tangent to $\gamma$. In this system $x=y=\left(0, y_{2}\right)$. We denote $\gamma_{\delta}=\gamma \cap B(t, \delta)$. We assume that $\gamma$ is $C^{3}$, then $\gamma_{\delta}$ has the parametrization $z_{2}=c z_{1}^{2}+O\left(z_{1}^{3}\right),\left|z_{1}\right|<\delta_{1} \leq \delta$.

Proposition 3.3 With the previous notations we have following asymptotic expansion as $y_{2} \rightarrow 0$ :

\section{Proof:}

$$
\mathcal{G}^{(s)}(y, y)=-\frac{1}{2 \pi^{2}} \log ^{2}\left(y_{2}\right)+O\left(\log \left(y_{2}\right)\right)
$$

Since $G_{r}(x, y)$ and $G_{t}(x, y)$ are regular (see Appendix A1), from (3.1) we have

$$
\mathcal{G}^{(s)}(y, y)=2 \int_{\gamma_{\delta}} G_{0}\left(k^{+}, y, z\right) \frac{\partial G_{0}\left(k^{+}, y, z\right)}{\partial n(z)} d s(z)+O(1),
$$


uniformly for $0<y_{2}<\delta$. Using the asymptotic formulas of Lemma 3.2, it follows when $y_{2} \rightarrow 0$ that

$$
\mathcal{G}^{(s)}(y, y)=\frac{1}{2 \pi^{2}} \int_{\gamma_{\delta}}\left(\frac{C}{|z-y|}+\frac{\log |z-y|}{|z-y|}\right) d s(z)+O(1) .
$$

If $\gamma$ is $C^{3}$, we have proved in the appendix A2 that

$$
\int_{\gamma_{\delta}} \frac{d s(z)}{|z-y|}=O\left(\log \left(y_{2}\right)\right), \quad \int_{\gamma_{\delta}} \frac{\log |z-y|}{|z-y|} d s(z)=-\log ^{2}\left(y_{2}\right)+O\left(\log \left(y_{2}\right)\right) .
$$

which ends the proof.

\section{Inverse Problem}

The inverse problem we consider is the following:

Given the wave numbers $k^{+}$and $k^{-}\left(k^{+} \neq k^{-}\right)$and the far field pattern $u_{\infty}(\theta, \phi):=$ $\left(A^{+}(\theta, \phi), A^{-}(\theta, \phi)\right)$ for all $\theta, \phi \in[-\pi, \pi]$, determine the interface $\gamma$.

\subsection{Uniqueness theorem}

We first introduce the following notations. For two functions $f_{j} \in C(\mathbb{R}) \cap C^{2}([-a, a])$, with supp $f_{j} \subset[-a, a], j=1,2$, let us consider the $\operatorname{arcs} \gamma_{j}:=\left\{\left(x_{1}, f_{j}\left(x_{1}\right)\right),-a<x_{1}<\right.$ $a\}$ and the domains $\Omega_{j}^{+}=\left\{\left(x_{1}, x_{2}\right) \in \mathbb{R}^{2}: x_{2}>f_{j}\left(x_{1}\right)\right\}$ and $\Omega_{j}^{-}=\left\{\left(x_{1}, x_{2}\right) \in \mathbb{R}^{2}: x_{2}<\right.$ $\left.f_{j}\left(x_{1}\right)\right\}$.

We prove the following uniqueness result.

Theorem 4.1 If the far field pattern $u_{\infty}^{(1)}(\theta, \phi)$ and $u_{\infty}^{(2)}(\theta, \phi)$ corresponding to $\gamma_{1}$ and $\gamma_{2}$ respectively, coincide for all $\theta, \phi \in[-\pi, \pi]$, then $\gamma_{1}=\gamma_{2}$.

\section{Proof:}

The proof will be done in three steps.

Step 1. Denote $D=\Omega_{1}^{+} \cap \Omega_{2}^{+}=\left\{\left(x_{1}, x_{2}\right) ; x_{2}>\max \left(f_{1}\left(x_{1}\right), f_{2}\left(x_{1}\right)\right)\right\}$. For an incident plane wave $u^{i n c}$ (which depends on $\theta$ ) the scattered waves $u_{1}$ and $u_{2}$ associated to $\gamma_{1}$ and $\gamma_{2}$ respectively coincide by Theorem 2.6.

Step 2. We now show that scattered waves $u_{1}(x, y)$ and $u_{2}(x, y)$ also coincide for an incident wave of the form $u^{i n c}(x, y)=G_{0}\left(k^{+}, x, y\right)$ with $y \in D$. In fact $u_{j}(x, y)=$ $\mathcal{G}_{j}^{(s)}(x, y)$ is the perturbed Green function corresponding to $\gamma_{j}$ (see section 3.1), then we conclude by combining step 1 with the reciprocity Theorem 3.1.

Step 3. Let us now assume that $\gamma_{1} \neq \gamma_{2}$. We can find a point $t \in \gamma_{1}$ and $\delta>0$ such that $B(t, \delta) \cap \Omega_{2}^{-}=\emptyset$. For $p \geq 1$, we consider the sequence $y_{p}=t+\frac{1}{p} n(t)$ which lies in $D$ for all $p \geq 1$. Let $u_{1}^{p}$ and $u_{2}^{p}$ be the solutions of the scattering problem (2.5) corresponding to $\gamma_{1}$ and $\gamma_{2}$ respectively, with the data $g_{j}^{p}=-\left[G\left(., y_{p}\right)\right]_{\gamma_{j}}$ and $h_{j}^{p}=-\left[\frac{\partial G\left(., y_{p}\right)}{\partial n}\right]_{\gamma_{j}}$. From step $2, u_{1}^{p}(x)=u_{2}^{p}(x)$ for $x \in D$. When $p \rightarrow+\infty$ we have by Proposition 3.3

$$
u_{1}^{p}\left(y_{p}\right) \simeq \frac{-1}{2 \pi^{2}} \log ^{2}(p) \rightarrow-\infty
$$


This is a contradiction with $u_{1}^{p}\left(y_{p}\right)=u_{2}^{p}\left(y_{p}\right)$ for all $p \geq 1$. Indeed the sequence $u_{2}^{p}$ converges uniformly in $H^{2}\left(B\left(t, \frac{\delta}{2}\right)\right)$ to $u_{2}(t, t)$, since $B(t, \delta) \cap \Omega_{2}^{-}=\emptyset$ and the boundary data $\left(g_{2}^{p}, h_{2}^{p}\right)$ converge in $\tilde{H}^{\frac{1}{2}}\left(\gamma_{2}\right) \times \tilde{H}^{-\frac{1}{2}}\left(\gamma_{2}\right)$. Hence $\gamma_{1}=\gamma_{2}$ and the proof is completed.

Remark 4.2 This method uses the fact that the scattered wave becomes singular at the boundary as the source point approach a boundary point. It has been employed by Kirsch and Kress for the transmission problem [15] and Hähner for isotropic medium (see [5, 8] and references therein). Our proof is based on the blow up of the scattered part of the Green function (Proposition 3.3) and the reciprocity relation (Proposition 3.1). So we have generalized the technique of point source (already used for bounded obstacle) to unbounded obstacle (namely $\Omega^{-}$).

\subsection{Reconstruction algorithm}

In this section, we use for simplicity the notation $\left(\mathcal{F}, u_{\infty}\right)$ instead of $\left(\mathcal{F}^{+}, u_{\infty}^{+}\right)$.

4.2.1. Iterative method To reconstruct the profile $\gamma$ from the far field measurement $u_{\infty}(\theta, \phi), \phi \in(0, \pi)$, we solve the non-linear operator equation

$$
\mathcal{F}(f)=u_{\infty}
$$

where $\mathcal{F}: D(\mathcal{F}) \subset X \rightarrow Y$ operates between the Hilbert spaces $\mathrm{X}$ and $\mathrm{Y}$ and is defined by $A^{+}(\theta, \phi)$ for $f \in D(\mathcal{F})$ (see $(2.12)$ ). In our application we choose $D(\mathcal{F})=H_{0}^{2}(-a, a)$, $X=H_{0}^{1}(-a, a)$ and $Y=L^{2}(] 0, \pi[)$.

To solve (4.2) we use a Newton-type algorithm: in each iteration step we solve the linear equation

$$
\mathcal{F}\left(f_{n}\right)+\mathcal{F}^{\prime}\left(f_{n}\right) h_{n}=u_{\infty}
$$

and then update $f_{n}$ by $f_{n+1}=f_{n}+h_{n}$ with an initial guess $f_{0} \in D(\mathcal{F})$.

Since $K_{n}:=\mathcal{F}^{\prime}\left(f_{n}\right)$, the Fréchet derivative of $\mathcal{F}$, is a compact operator (see remark 4.4), then (4.3) is ill-posed, the solution does not depend continuously on the given data. So, if we have perturbed data $u_{\infty}^{\delta}$ instead of $u_{\infty}$ in (4.2) satisfying $\left\|u_{\infty}^{\delta}-u_{\infty}\right\| \leq \delta$, then we need to regularize the problem in order to compute approximate solutions $f^{\delta}$ that converge to some solution of (4.2) as $\delta \rightarrow 0$. Tikhonov regularization is certainly the most well-known regularization method but in our case we propose a better method to the linear system (4.3). As inner iteration we apply a few steps of the conjugate gradient method (CG) to the normal equation associated with (4.3):

$$
K_{n}^{*} K_{n} h_{n}=K_{n}^{*}\left(\mathcal{F}\left(f_{n}\right)-u_{\infty}\right) .
$$

The Matlab function lsqr from the Regularization Tools program package by Hansen (see [11]) implements the CG algorithm applied to normal equation (4.4). The outer iteration (4.3) is terminated at the first index $n(\delta)$ for which the residual in less than the noise level $\delta$, that is,

$$
\left.\| \mathcal{F}\left(f_{n(\delta)}\right)-u_{\infty}^{\delta}\right) \| \leq \tau \delta
$$


with some parameter $\tau>1$.

Hanke (see [13]) has proved the convergence of the CG-method under the following assumption on the nonlinearity of $\mathcal{F}$ :

$$
\left\|\mathcal{F}\left(f_{2}\right)-\mathcal{F}\left(f_{1}\right)-\mathcal{F}^{\prime}\left(f_{1}\right)\left(f_{2}-f_{1}\right)\right\| \leq C\left\|f_{2}-f_{1}\right\|\left\|\mathcal{F}\left(f_{2}\right)-\mathcal{F}\left(f_{1}\right)\right\| .
$$

Unfortunately, this condition is not yet verified in our situation.

4.2.2. Fréchet derivative From now, we assume that the incident angle $\theta$ is fixed and the observation angle $\phi \in(0, \pi)$. The equation $(2.12)$ can be written

$$
\left.\mathcal{F}(f)(\phi)=u_{\infty}(\phi), \quad \phi \in\right] 0, \pi[,
$$

where the non linear integral operator is given by:

$$
\left.\mathcal{F}(f)(\phi)=\int_{-a}^{a} F^{+}\left(\theta, \phi, y_{1}, f\left(y_{1}\right), f^{\prime}\left(y_{1}\right)\right) d y_{1}, \quad \phi \in\right] 0, \pi[,
$$

with

$$
F^{+}\left(\theta, \phi, y_{1}, f\left(y_{1}\right), f^{\prime}\left(y_{1}\right)=F_{1}^{+}\left(\theta, \phi, y_{1}, f\left(y_{1}\right), f^{\prime}\left(y_{1}\right)\right)+S_{r}(\phi) F_{2}^{+}\left(\theta, \phi, y_{1}, f\left(y_{1}\right), f^{\prime}\left(y_{1}\right)\right)\right.
$$

and

$$
\begin{aligned}
F_{1}^{+}\left(\theta, \phi, y_{1}, f\left(y_{1}\right), f^{\prime}\left(y_{1}\right)\right) & =\left(A_{1} f^{\prime}\left(y_{1}\right)+B_{1}\right) \exp \left(-A_{1} y_{1}-B_{1} f\left(y_{1}\right)\right) \\
& +R(\theta)\left(A_{1} f^{\prime}\left(y_{1}\right)+B_{2}\right) \exp \left(A_{1} y_{1}+B_{2} f\left(y_{1}\right)\right) \\
& +T(\theta)\left(-A_{1} f^{\prime}\left(y_{1}\right)+B_{3}\right) \exp \left(-A_{1} y_{1}-B_{3} f\left(y_{1}\right)\right), \\
F_{2}^{+}\left(\theta, \phi, y_{1}, f\left(y_{1}\right), f^{\prime}\left(y_{1}\right)\right) & =\left(A_{1} f^{\prime}\left(y_{1}\right)-B_{2}\right) \exp \left(A_{2} y_{1}-B_{1} f\left(y_{1}\right)\right) \\
& +R(\theta)\left(A_{1} f^{\prime}\left(y_{1}\right)+B_{1}\right) \exp \left(A_{2} y_{1}+B_{2} f\left(y_{1}\right)\right) \\
& +T(\theta)\left(-A_{1} f^{\prime}\left(y_{1}\right)+B_{4}\right) \exp \left(A_{1} y_{1}-B_{3} f\left(y_{1}\right)\right) .
\end{aligned}
$$

The coefficients are defined in (2.13) and (2.15).

Proposition 4.3 We assume that $f_{0} \in H_{0}^{2}(-a, a)$. The Fréchet derivative $K_{0}=\mathcal{F}^{\prime}\left(f_{0}\right)$ is the linear integral operator $K_{0}: H_{0}^{1}(-a, a) \rightarrow L^{2}(] 0, \pi[)$ defined by:

$$
K_{0} h(\phi)=\int_{-a}^{a} k\left(\phi, y_{1}\right) h\left(y_{1}\right) d y_{1}
$$

with the kernel

$$
k\left(\phi, y_{1}\right)=F_{f}-F_{y_{1} f^{\prime}}-f_{0}^{\prime}\left(y_{1}\right) F_{f f^{\prime}} \quad \text { at the point }\left(\phi, y_{1}, f_{0}\left(y_{1}\right), f_{0}^{\prime}\left(y_{1}\right)\right)
$$

\section{Proof:}

The mean value theorem gives

$$
F\left(\phi, y_{1}, f_{0}+h, f_{0}^{\prime}+h^{\prime}\right)=F\left(\phi, y_{1}, f_{0}, f_{0}^{\prime}\right)+h F_{f}+h^{\prime} F_{f^{\prime}}+o\left(|h|+\left|h^{\prime}\right|\right) .
$$


This implies

$$
\begin{aligned}
\mathcal{F}\left(f_{0}+h\right) & =\mathcal{F}\left(f_{0}\right)+\int_{-a}^{a}\left(h F_{f}+h^{\prime} F_{f^{\prime}}\right) d y_{1}+o\left(\|h\|_{L^{2}}+\left\|h^{\prime}\right\|_{L^{2}}\right) \\
& =\mathcal{F}\left(f_{0}\right)+\int_{-a}^{a}\left(h F_{f}-h \frac{d F_{f^{\prime}}}{d y_{1}}\right) d y_{1}+o\left(\|h\|_{H^{1}}\right) \\
& =\mathcal{F}\left(f_{0}\right)+\int_{-a}^{a}\left(F_{f}-F_{f f^{\prime}} f_{0}^{\prime}-F_{y_{1} f^{\prime}}-f_{0}^{\prime \prime} F_{f^{\prime} f^{\prime}}\right) h d y_{1}+o\left(\|h\|_{H^{1}}\right) \\
& =\mathcal{F}\left(f_{0}\right)+\int_{-a}^{a}\left(F_{f}-F_{f f^{\prime}} f_{0}^{\prime}-F_{y_{1} f^{\prime}}\right) h d y_{1}+o\left(\|h\|_{H^{1}}\right) .
\end{aligned}
$$

Note that $F_{f^{\prime} f^{\prime}}=0$ since the mapping $f^{\prime} \rightarrow F$ is linear.

More precisely, the kernel $k\left(\phi, y_{1}\right)$ is given by the following expression:

$$
k\left(\phi, y_{1}\right)=k_{1}\left(\phi, y_{1}\right)+S_{r}(\phi) k_{2}\left(\phi, y_{1}\right)
$$

with

$$
\begin{aligned}
k_{1}\left(\phi, y_{1}\right) & =K_{1} \exp \left(-A_{1} y_{1}-B_{1} f_{0}\left(y_{1}\right)\right)+R(\theta) K_{2} \exp \left(A_{1} y_{1}+B_{2} f_{0}\left(y_{1}\right)\right) \\
& +T(\theta) K_{3} \exp \left(-A_{1} y_{1}-B_{3} f_{0}\left(y_{1}\right)\right), \\
k_{2}\left(\phi, y_{1}\right)= & K_{4} \exp \left(A_{2} y_{1}-B_{1} f_{0}\left(y_{1}\right)\right)+T(\theta) K_{5} \exp \left(A_{1} y_{1}-B_{3} f_{0}\left(y_{1}\right)\right)
\end{aligned}
$$

and

$$
\begin{aligned}
& K_{1}=-B_{1}^{2}+A_{1}^{2}=-2\left(k^{+}\right)^{2} \cos (\theta+\phi)[\cos (\theta-\phi)+1], \\
& K_{2}=B_{2}^{2}-A_{1}^{2}=2\left(k^{+}\right)^{2} \cos (\theta-\phi)[\cos (\theta+\phi)+1], \\
& K_{3}=-B_{3}^{2}-A_{1}^{2}=\left(k^{+}\right)^{2}+\left(k^{-}\right)^{2}+2\left(k^{+}\right)^{2} \cos \theta \cos \phi+2 k^{+} \sin \phi \sqrt{\left(k^{-}\right)^{2}-\left(k^{+}\right)^{2} \cos ^{2} \theta}, \\
& K_{4}=-B_{1} B_{2}-A_{1} A_{2}=2\left(k^{+}\right)^{2} \sin (\theta-\phi) \sin (\theta+\phi), \\
& K_{5}=-B_{3} B_{4}+A_{1}^{2}=k^{+}(\sin \theta+\sin \phi)\left(k^{-} \sin \chi+k^{+} \sin \phi\right)-\left(k^{+}\right)^{2}(\cos \theta+\cos \phi)^{2}, \\
& \quad R(\theta)=\frac{a_{2}-c_{1}}{a_{2}+c_{1}}, \quad T(\theta)=\frac{2 a_{2}}{a_{2}+c_{1}}, \quad S_{r}(\phi)=\frac{-i b_{2}-\sqrt{\left(k^{-}\right)^{2}+b_{1}^{2}}}{-i b_{2}+\sqrt{\left(k^{-}\right)^{2}+b_{1}^{2}}} .
\end{aligned}
$$

The coefficients $A_{j}$ and $B_{j}$ are defined in (2.13) and (2.15).

\section{Remark 4.4}

1) If $f_{0} \in H_{0}^{2}(-a, a), K_{0}$ is in fact a bounded operator in $L^{2}(] 0, \pi[)$ and is compact (the kernel $k\left(\phi, y_{1}\right)$ is a bounded function).

2) If $f_{0} \in H_{0}^{1}(-a, a)$ then $K_{0}: H_{0}^{1}(-a, a) \rightarrow L^{2}(] 0, \pi[)$ is bounded (but not compact) and given by:

$$
K_{0} h=\int_{-a}^{a}\left(h\left(y_{1}\right) F_{f}\left(\phi, y_{1}, f_{0}, f_{0}^{\prime}\right)+h^{\prime}\left(y_{1}\right) F_{f^{\prime}}\left(\phi, y_{1}, f_{0}, f_{0}^{\prime}\right)\right) d y_{1} .
$$




\section{Numerical Results}

In this last section, we give some numerical tests of the proposed method. These numerical results allow us to underline the effects of some parameters, such as the incident angle, the geometry or the noise, on the reconstruction. The reconstruction is obtained from the far field pattern for a single illumination by a plane wave at a fixed frequency. In all the examples we determine the shape of a one-dimensional interface between two half-spaces. The method converges rapidly and yields highly resolved reconstructions after 20 iterations. In the figure 2 we present the reconstruction of rough interface between two dielectric half-spaces and in the figure 3-4-5 the reconstruction of different underwater profiles. The examples are presented for incidence directions $\theta=\pi / 2$ and $\theta=\pi / 6$ without noise. Note that the reconstruction is not sensitive to noise of about $5 \%$. Obviously, the best reconstruction is obtained for the case of normal incidence. In all examples we used $N=160$ points in the discretization of the parametrization $f$. The synthetic data for the far field $u_{\infty}(\theta, \phi)$ were obtained via trapezoidal quadrature rule with $N$ collocation points $\phi_{j}=\frac{j \pi}{N}, j=1$ to $N$. The CG-algorithm is stopped after three steps. We note that if we continue the process the precision is deteriorated. This phenomena is pointed out in the literature concerning the regularization of the nonlinear ill-posed problems $([13,12])$ and the stopping rule in the inner or outer iterations is called the generalized discrepancy principle.

In conclusion, the numerical results show accurate reconstruction when the size of the roughness $H$ is less than $0.3 \lambda$ where $\lambda$ is the wavelength. The algorithm is stable with respect to data with a noise of about $5 \%$. For $H \geq 0.5 \lambda$ the construction starts to deteriorate (see figure 5). Indeed, for this range of frequencies, the nonlinear equation if severely ill-posed in the sense that the linearized equation is ill-conditioned. The performance of the conjugate gradient method can be improved by the use of adequate pre-conditioner $([6])$, hence further research is required to expect better reconstruction for such a profile. 
(i) Reconstruction of dielectric profile with the parameters $\lambda=0.632, k^{+}=\frac{2 \pi}{\lambda}$, $k^{-}=1.5 k^{+}$for incidence angles $\theta=\frac{\pi}{2}$ (left) and $\theta=\frac{\pi}{6}$ (right). Here we have an interface between air and glass with trapezoidal shape (depth $H=0.1 \mu m$, base $b=4 \mu m)$.
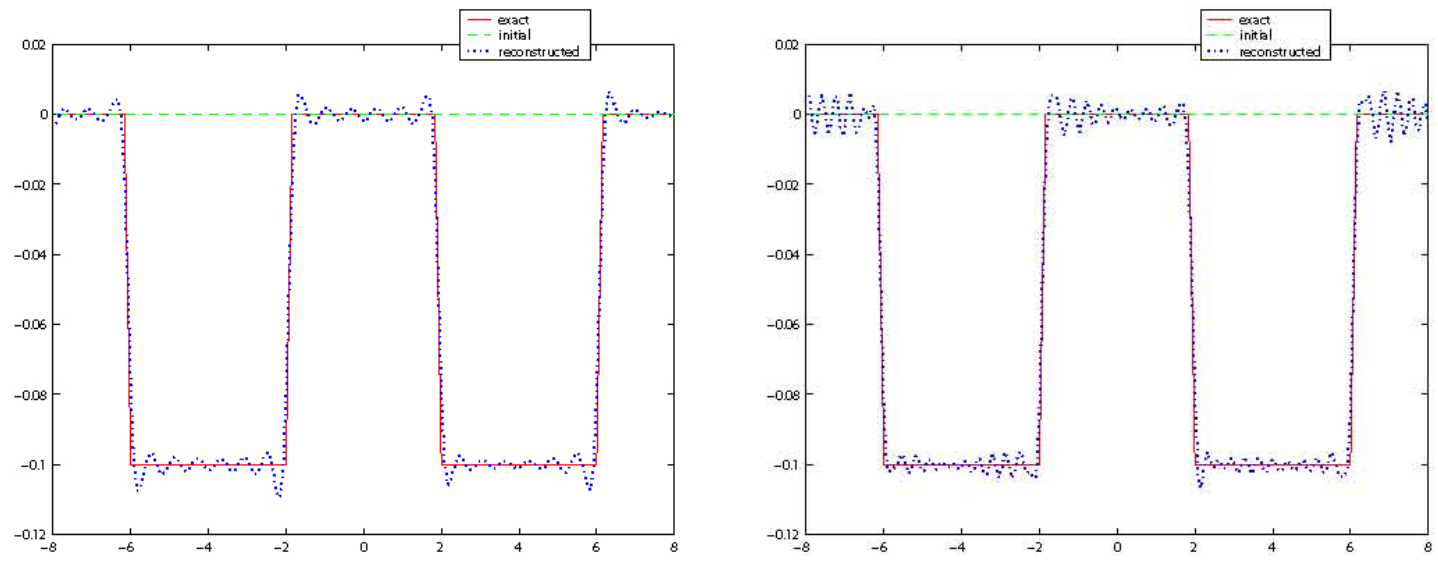

Figure 2

(ii) Reconstruction of underwater profiles with the parameters $k^{+}=4, k^{-}=1.5 k^{+}$, $H=0.3$ and $\theta=\frac{\pi}{2}$. On the right, we plot the rate of the convergence.
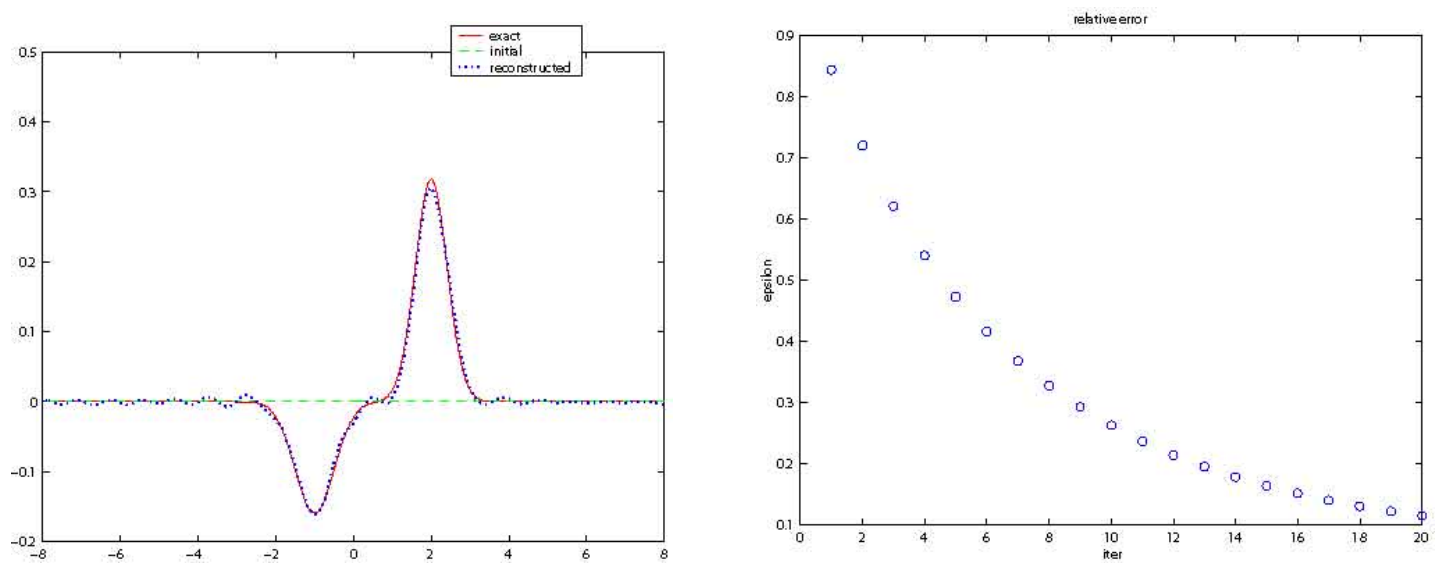

Figure 3 
(iii) Reconstruction of underwater profiles with the parameters $k^{+}=4, k^{-}=1.5 k^{+}$, $H=0.3$ and $\theta=\frac{\pi}{6}$. On the right, we show the scattering diagram.
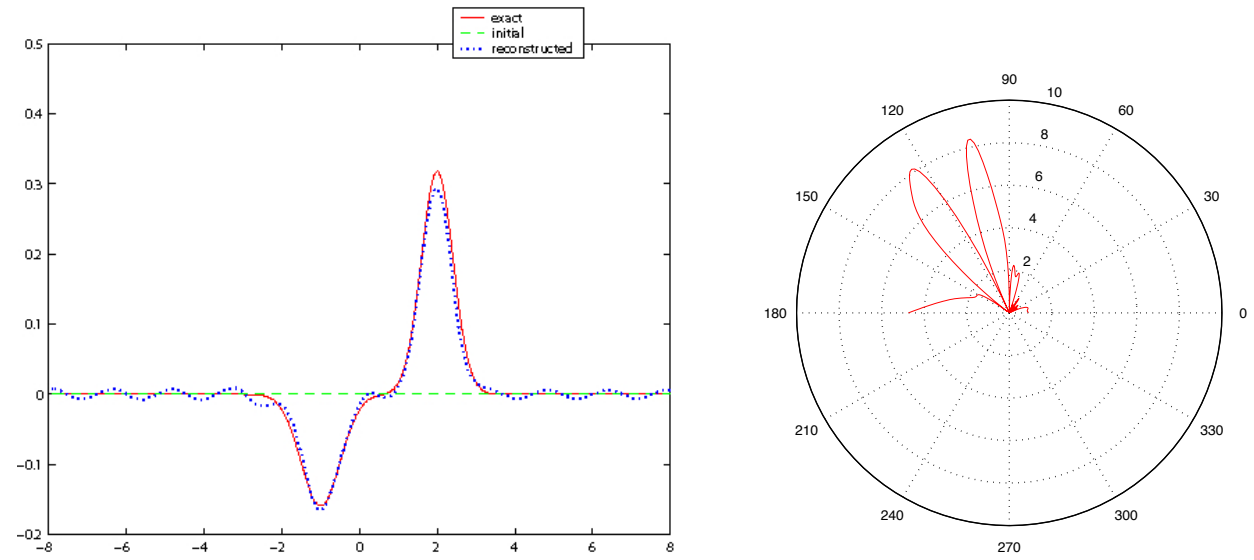

Figure 4

(iv) Reconstruction of underwater profiles with the parameters $k^{+}=4, k^{-}=1.5 k^{+}$, $H=0.5$ and $\theta=\frac{\pi}{2}$. On the right, we plot the rate of the convergence.
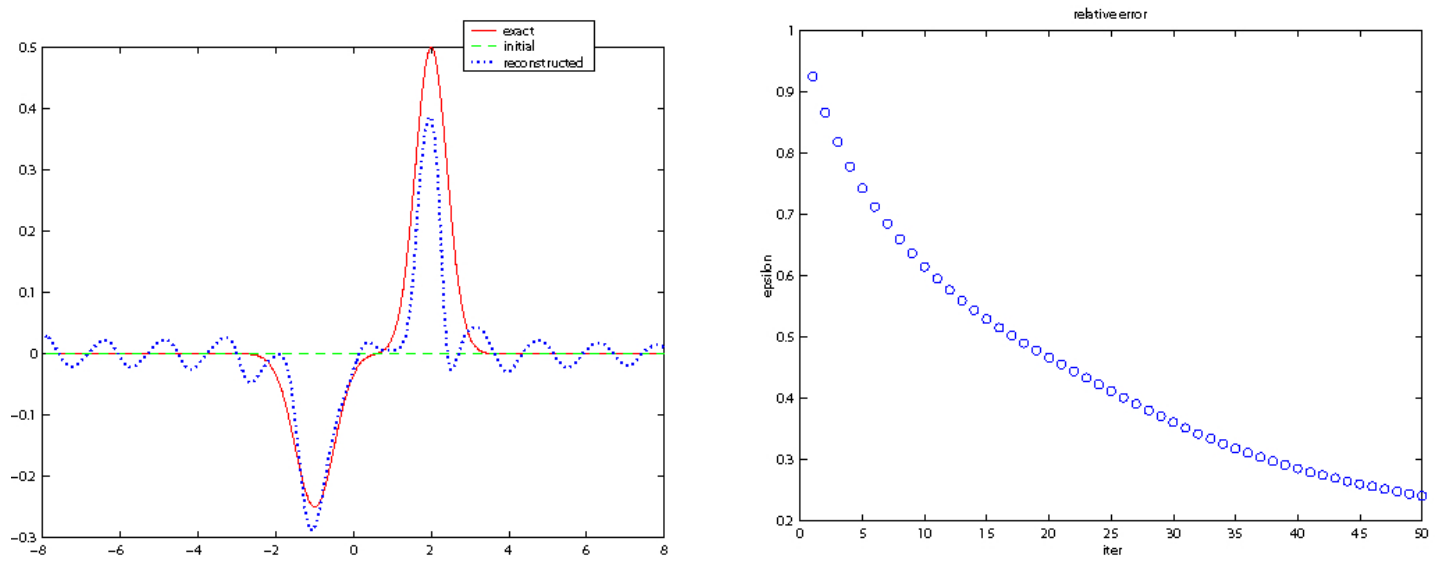

Figure 5 
Reconstruction of the interface between two layered media

\section{Appendix}

\section{A1. Green's function $G(x, y)$}

Let $G(x, y)$ be the outgoing Green's function of the unperturbed two-half-spaces, that is, the solution of

$$
\begin{gathered}
\left(\Delta_{x}+k^{ \pm}\right) G(x, y)=\delta(x-y) \text { in } \mathbb{R}_{ \pm}^{2} \quad\left(y_{2} \neq 0\right), \\
{[G(x, y)]=\left[\frac{\partial G(x, y)}{\partial x_{2}}\right]=0 \text { on } x_{2}=0}
\end{gathered}
$$

with, in each domain, the Sommerfeld radiation condition as $r=|x| \rightarrow+\infty$

$$
\lim _{r \rightarrow+\infty} \sqrt{r}\left(\frac{\partial G}{\partial r}-i k^{ \pm} G\right)=0
$$

We refer to [10] for the existence and the uniqueness of $G$. If $y_{2}>0$, by construction, $G$ is decomposed into $G(x, y)=G_{0}\left(k^{+}, x, y\right)+G_{r}(x, y)$ for $x_{2}>0$ and $G(x, y)=G_{t}(x, y)$ for $x_{2}<0$, where $G_{r}$ and $G_{t}$ solve Helmholtz equation in the whole space $\mathbb{R}^{2}$ and satisfy the transmission conditions

$$
G_{r}-G_{t}=-\left[G_{0}\right], \quad \frac{\partial}{\partial x_{2}}\left(G_{r}-G_{t}\right)(x, y)=-\left[\frac{\partial G_{0}(x, y)}{\partial x_{2}}\right] \text { on } x_{2}=0 .
$$

Then $G_{r}$ and $G_{t}$ are analytic functions in $\mathbb{R}^{2}$. However $G$ is (globally) in $C^{1}\left(\mathbb{R}^{2}\right)$ by elliptic regularity.

\section{A2. Asymptotic expansion}

We suppose that $\gamma$ is $C^{3}$ and put $\gamma_{\delta}=\gamma \cap B(0, \delta)$, then $\gamma_{\delta}=\left\{\left(t, c t^{2}+O\left(t^{3}\right)\right),|t| \leq \delta\right\}$. Let $y=(0, \eta)$, with $\eta>0$. Our aim is to estimate the following integrals when $\eta \rightarrow 0$

$$
I_{1}(\eta)=\int_{\gamma} \frac{d s(z)}{|y-z|}, \quad I_{2}(\eta)=\int_{\gamma} \frac{\log |y-z|}{|y-z|} d s(z) .
$$

From $|y-z|^{2}=\eta^{2}+(1-2 c \eta) t^{2}+O\left(t^{3}\right)=\rho^{2}(t, \eta)+O\left(t^{3}\right)$, we deduce

$$
\frac{1}{|y-z|}=\frac{1}{\rho}+O(1), \log |y-z|=\log \rho+O(t) \text { and } \frac{\log |y-z|}{|y-z|}=\frac{\log \rho}{\rho}+O(\log (t)) \text {. }
$$

Then

$$
I_{1}(\eta)=2 \int_{0}^{\delta} \frac{d t}{\rho(t, \eta)}+O(1) \text { and } I_{2}(\eta)=2 \int_{0}^{\delta} \frac{\log \rho(t, \eta)}{\rho(t, \eta)} d t+O(1) .
$$

If we set $u=\frac{\sqrt{1-2 c \eta}}{\eta} t$, we obtain

$$
\int_{0}^{\delta} \frac{d t}{\rho(t, \eta)}=\frac{1}{\sqrt{1-2 c \eta}} \int_{0}^{A(\eta)} \frac{d u}{\sqrt{1+u^{2}}}
$$

and

$$
\int_{0}^{\delta} \frac{\log \rho(t, \eta)}{\rho(t, \eta)} d t=\frac{\log (\eta)}{\sqrt{1-2 c \eta}} \int_{0}^{A(\eta)} \frac{d u}{\sqrt{1+u^{2}}}+\frac{1}{\sqrt{1-2 c \eta}} \int_{0}^{A(\eta)} \frac{\log \sqrt{1+u^{2}}}{\sqrt{1+u^{2}}} d u
$$


where $A(\eta)=\frac{\delta \sqrt{1-2 c \eta}}{\eta} \simeq \frac{\delta}{\eta}$.

We obtain when $\eta \rightarrow 0$

$$
\int_{0}^{A(\eta)} \frac{d u}{\sqrt{1+u^{2}}}=-\log \eta+O(1)
$$

and

$$
\int_{0}^{A(\eta)} \frac{\log \sqrt{1+u^{2}}}{\sqrt{1+u^{2}}} d u=\int_{1}^{A(\eta)} \frac{\log u}{u} d u+O(1)=\frac{1}{2} \log ^{2} \eta+O(1) .
$$

Finally we get $I_{1}(\eta)=-2 \log \eta+O(1)$ and $I_{2}(\eta)=-\log ^{2} \eta+O(1)$.

Acknowledgments. This research was finalized when the first author is visiting the LATP (Laboratory of the Aix-Marseille University) during the second semester 2010. We would like to thank Professor Yves Dermenjian for useful discussions.

\section{References}

[1] M. Abramowitz And I.A. Stegun, Handbook of Mathematical Functions, National Bureau of Standards. Appl. math. Series 55, 1972.

[2] I. Akduman, R. Kress And A. Yapar, Iterative reconstruction of dielectric rough surface profiles at fixed frequency, Inverse Problems 22 (2006), 939-954.

[3] A. Ardjouni, Etude mathématique de la diffraction dans un dioptre perturbé, Mémoire de Magister, Université de Annaba (Algérie), 2004.

[4] F. Cakoni and D. Colton, The linear sampling method for cracks, Inverse Problems 19 (2003).

[5] F. Cakoni and D. Colton, Qualitative Methods in Inverse Scattering Theory, IMM, SpringerVerlag, 2006.

[6] D. Calvetti, L. Reichel And A. Shuibi, Invertible smoothing preconditioners for linear discrete ill-posed problems, Appl. Numer. Math. 54 13549, 2005.

[7] D. Colton and R. KRess, Inverse acoustic and electromagnetric Scattering Theory, Appl. Math. Sci., 93, Berlin-Springer, 1998.

[8] D. Colton And R. Kress, Using fundamental solutions in inverse problems, Inverse Problems 22 (2006), 49-46.

[9] J. CoYle, Locating the support of object contained in a two layered background medium in two dimensions, Inverse Problems 16 (2000).

[10] J.A. DeSanto, Scalar Wave Theory. Green's Functions and Applications, Springer Series on Wave Phenomena, Springer-Verlag, Berlin, 1992.

[11] P.C. Hansen, Regularisation tools. A Matlab package for analysis and solution for discrete illposed problems, http://www.imm.dtu.dk/ pch, Mars 2008.

[12] M. Hanke, A. Neubauer, O. Scherzer, Convergence analysis of the Landweber iteration for nonlinear ill-posed problems, Numer. Math. , 72, (1995), 21:37.

[13] M. Hanke, Regularizing properties of a truncated Newton-CG algorithm for nonlinear inverse problems, Numer. Funct. Anal. Optim., Vol. 18, No. 9-10 (1997), 971:993.

[14] E. IAkovliva, Inverse Scattering from Small Inhomogeneities, Ph.D. Thesis, Ecole Polytechnique , 2004.

[15] A. Kirsch And R. Kress, Uniqueness in inverse scattering problems, Inverse Problems 9 (1993), 285-299. 
[16] R. KREss , Newton's method for inverse obstacle scattering meets the method of least squares, Inverse Problems 19 (2003), 91-104.

[17] R. KRess And W. Rundell, A quasi-Newton method in inverse scattering, Inverse Problems 10 (1994), 1145-157.

[18] R. Kress and P. Serranho, A hybrid method for two-dimensional crack reconstruction, Inverse Problems 21 (2005), 773-784.

[19] R. Kress , N. Tezel and F. Yaman, A second order Newton method for sound soft inverse obstacle scattering, Journal of Inverse and Ill-Posed Problems 17 (2009), 173-185.

[20] G. Kristensson, Uniqueness theorems for the Helmholtz equation: penetrable media with an infinite interface, SIAM J. Math. Anal., Vol. 11, No. 6, (1980).

[21] F. M. ODEH, Uniqueness theorems for the Helmholtz equation in domains with infinite boundaries, Mathematics and Mechanics, Vol. 16, No. 6, (1963). 